See discussions, stats, and author profiles for this publication at: https://www.researchgate.net/publication/327231949

\title{
Alkylating histone deacetylase inhibitors may have therapeutic value in experimental myeloperoxidase-ANCA vasculitis
}

Article in Kidney International · August 2018

DOI: 10.1016/j.kint.2018.05.028

\section{CITATIONS}

13 authors, including:

Dearbhaile Dooley

University College Dublin

28 PUBLICATIONS 320 CITATIONS

SEE PROFILE

Gareth Brady

Trinity College Dublin

24 PUBLICATIONS 2,153 CITATIONS

SEE PROFILE

Some of the authors of this publication are also working on these related projects:

Diabetic nephropathy View project

Role of LAMP-2 in tolerance to ANCA antigens View project
101

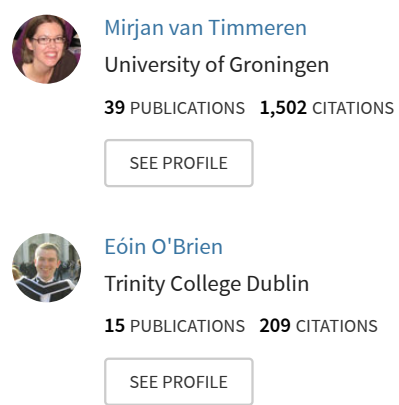




\title{
Alkylating histone deacetylase inhibitors may have therapeutic value in experimental myeloperoxidase-ANCA vasculitis
}

\author{
Dearbhaile Dooley ${ }^{1}$, Mirjan M. van Timmeren ${ }^{2}$, Vincent P. O’Reilly', Gareth Brady ${ }^{1}$, Eóin C. O’Brien ${ }^{1}$, \\ Barbara Fazekas ${ }^{1}$, Fionnuala B. Hickey ${ }^{1}$, Emma Leacy ${ }^{1}$, Charles D. Pusey ${ }^{3}$, Frederick W.K. Tam ${ }^{3}$, \\ Thomas Mehrling ${ }^{4}$, Peter Heeringa ${ }^{2}$ and Mark A. Little ${ }^{1,5}$
}

\begin{abstract}
${ }^{1}$ Trinity Health Kidney Centre, Trinity Translational Medicine Institute, Trinity College Dublin, St. James' Hospital Campus, Dublin, Ireland; ${ }^{2}$ Department of Pathology and Medical Biology, University Medical Center Groningen, University of Groningen, Groningen, The Netherlands; ${ }^{3}$ Renal and Vascular Inflammation Section, Department of Medicine, Imperial College London, London, UK; ${ }^{4}$ MundipharmaEDO GmbH, Basel, Switzerland; and ${ }^{5}$ rrish Centre for Vascular Biology, Trinity College Dublin, Dublin, Ireland
\end{abstract}

Current therapies for treating antineutrophil cytoplasm autoantibody (ANCA)-associated vasculitis include cyclophosphamide and corticosteroids. Unfortunately, these agents are associated with severe adverse effects, despite inducing remission in most patients. Histone deacetylase inhibitors are effective in rodent models of inflammation and act synergistically with many pharmacological agents, including alkylating agents like cyclophosphamide. EDO-S101 is an alkylating fusion histone deacetylase inhibitor molecule combining the DNA alkylating effect of Bendamustine with a pan-histone deacetylase inhibitor, Vorinostat. Here we studied the effects of EDO-S101 in two established rodent models of ANCA-associated vasculitis: a passive mouse model of antimyeloperoxidase IgG-induced glomerulonephritis and an active rat model of myeloperoxidase-ANCA microscopic polyangiitis. Although pretreatment with EDO-S101 reduced circulating leukocytes, it did not prevent the development of passive IgG-induced glomerulonephritis in mice. On the other hand, treatment in rats significantly reduced glomerulonephritis and lung hemorrhage. EDOS101 also significantly depleted rat B and T cells, and induced DNA damage and apoptosis in proliferating human $B$ cells, suggesting a selective effect on the adaptive immune response. Thus, EDO-S101 may have a role in treatment of ANCA-associated vasculitis, operating primarily through its effects on the adaptive immune response to the autoantigen myeloperoxidase.

Kidney International (2018) ש, घ-目; https://doi.org/10.1016/ j.kint.2018.05.028

KEYWORDS: albuminuria; ANCA; glomerulonephritis; inflammation; renal pathology

Copyright (c) 2018, International Society of Nephrology. Published by Elsevier Inc. All rights reserved.

\footnotetext{
Correspondence: Mark A. Little, Trinity Health Kidney Centre, Trinity Translational Medicine Institute, Trinity College Dublin, St. James' Hospital Campus, Dublin 8, Ireland. E-mail: mlittle@tcd.ie
}

Received 6 October 2017; revised 4 May 2018; accepted 24 May 2018
A ntineutrophil cytoplasm autoantibody (ANCA)-associated vasculitis (AAV) is a systemic autoimmune condition that affects small- to medium-sized blood vessels. Severe vessel wall damage causes inflammatory necrosis and consequent loss of organ function. The lungs and kidneys are frequently affected, leading to lung hemorrhage and glomerulonephritis (GN). Patients with AAV develop circulating autoantibodies against the neutrophil granule and monocyte lysosomal enzymes myeloperoxidase (MPO) or proteinase $3 .^{1,2}$ Conventional treatment includes cyclophosphamide and corticosteroids, which induces remission in most patients. However, these therapies do not fully prevent disease relapse and patients often require long-term treatment, which is associated with severe morbidity. ${ }^{3}$ This highlights the urgent need for the development of new therapies.

Histone deacetylase inhibitors (HDACis) were originally described as a class of anticancer drugs in 2006 because of their growth arrest and apoptotic effects on tumor cells. ${ }^{4}$ They target the histone deacetylase enzymes that remove the acetyl groups from lysine residues, leading to chromatin condensation and transcriptional silencing. HDACis thus result in histone hyperacetylation, thereby affecting gene transcription. This enhances activity of some transcription factors such as the tumor suppressor p53, but represses B- and T-cell transcription factors. Therefore, in the setting of autoimmunity, HDACis can induce cell cycle arrest or apoptosis of key leukocyte populations that are proliferating in response to autoantigen exposure. Recently, HDACis were shown to have beneficial effects in rodent models of inflammation, including arthritis, asthma, and colitis; however, their mechanisms of action are not well understood. ${ }^{5}$ HDACis act synergistically with a diverse range of pharmacological and biological agents, including cyclophosphamide. Therefore, it appears likely that HDACis may be best used in the clinic as part of a combination regimen, enabling a multitarget approach.

EDO-S101, a small molecule compound developed by Mundipharma-EDO $\mathrm{GmbH}$, is an alkylating HDACi fusion molecule that combines the strong DNA damaging effect of bendamustine with a fully functional pan-HDACi, vorinostat. This combination therapy has the potential to provide enhanced 
efficacy at a lower alkylating agent dose owing to its bifunctional mode of action. HDAC inhibition allows better access of the alkylating moiety to DNA double strands by opening the chromatin, thereby creating the potential for enhanced synergy between the 2 moieties. EDO-S101 has demonstrated good tolerability and exerts significant activity against hematological malignant neoplasia and solid tumors. ${ }^{6-8}$

We hypothesized that EDO-S101 is an effective therapy for AAV. To dissect its effects on both the innate and the adaptive immune response, we assessed its efficacy in 2 wellestablished rodent models of MPO-AAV: a passive mouse model of anti-MPO IgG-induced GN and an active rat model of experimental autoimmune vasculitis (EAV). EAV is an MPO-ANCA vasculitis model induced by immunization of Wistar Kyoto rats with anti-human MPO (hMPO), resulting in crescentic GN and lung hemorrhage. These features of mild pauci-immune vasculitis are indistinguishable pathologically from human vasculitis, making EAV a valuable preclinical model in AAV. We observed that EDO-S101 was largely ineffective in the murine model but dramatically improved renal and lung disease in the rat model. Thus, EDO-S101 has potential as a novel treatment for AAV, largely through its action on the adaptive immune response.

\section{RESULTS}

EDO-S101 induces global histone 3 hyperacetylation in HL60 cells and a strong DNA repair response in vivo

Hyperacetylation of the histone 3 tail at lysine residues K9, $\mathrm{K} 14, \mathrm{~K} 23$, and K56 was measured using specific antibodies for acetylated lysine residues in total cell extracts of HL60 cells (Figure 1a). EDO-S101 demonstrated enhanced acetylation of lysine residues, whereas bendamustine was equivalent to dimethylsulfoxide control (Figure 1a). Exposure to EDO-S101 in vivo caused a strong DNA repair response (activation of p53 and pH2AX) in tumor samples taken at days 4 and 8 from mice with subcutaneous human Daudi Burkitt lymphoma (Figure 1b). Both ataxia telangiectasia mutated (ATM) kinase and Rad3-related protein (ATR) are activated upon DNA damage. After their recruitment to DNA damage sites, ATM and ATR activate checkpoint kinase 2 and checkpoint kinase 1 , respectively. These kinases were investigated because their pathways are central in DNA damage repair and their overactivation may confer aggressive molecular features, including endogenous DNA damage and oncogene-induced replication stress. ${ }^{9}$ Taken together, these allowed us to investigate the simultaneous effects of EDOS101 on DNA damage and blocking of damage repair. Indirect HDACi activity was measured in tumor samples on days 4 and 8; phosphorylated (p)-ATR and phosphorylated (p)ATM were dose dependently suppressed by EDO-S101 treatment. In contrast, phosphorylated (p)-CHK2 was strongly upregulated at day 4 , but returned to undetectable levels at day 8 (Supplementary Figure S1A).

\section{EDO-S101 has little effect on anti-MPO-induced vasculitis in a passive transfer model of MPO-AAV}

Given the potent combination of HDACi and alkylating capacity of the compound, we assessed the ability of EDO-S101 to prevent vasculitic injury induced by anti-MPO antibodies in a passive transfer model, with the compound given from the time of antibody transfer. Circulating anti-MPO antibody levels were similar in vehicle- and EDO-S101-pretreated mice at day 1 and day 7 (Figure 2a; Supplementary Figure S2A). After disease induction, both vehicle- and EDO-S101-pretreated mice developed characteristic hematuria, which decreased over time (from day 1 to day 7). Although there was no significant effect on hematuria (Supplementary Figure S2B), EDO-S101 reduced albuminuria than did vehicle (Figure 2b). Both vehicle- and EDO-S101-pretreated mice developed GN (Figure $2 \mathrm{~d}-\mathrm{k}$ ), with no significant effect of EDO-S101 on crescentic GN (Figure 2c) or glomerular histopathology evident (Supplementary Figure S3A and B).

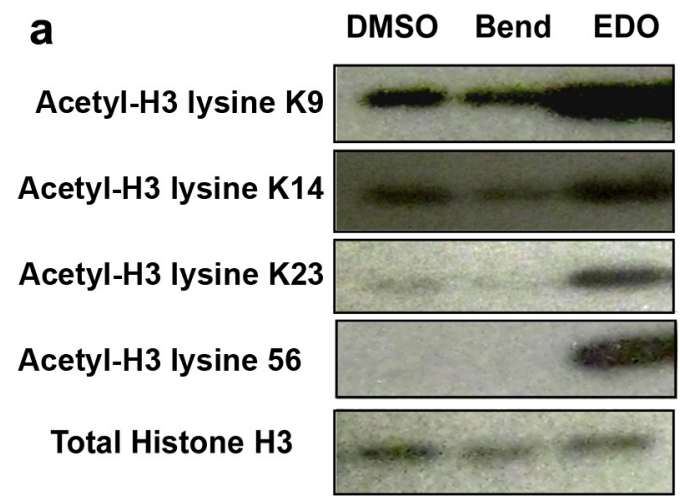

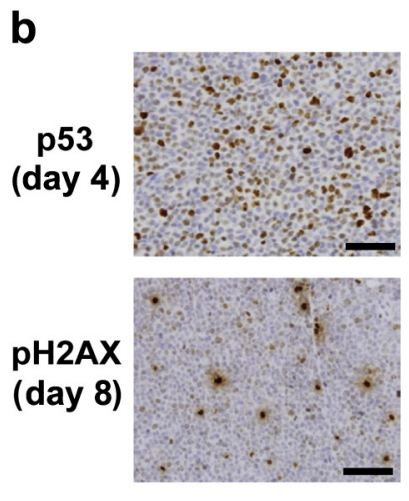

Vehicle
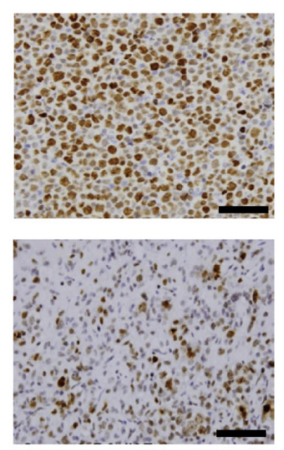

EDO (40 $\mathrm{mg} / \mathrm{kg}$ )
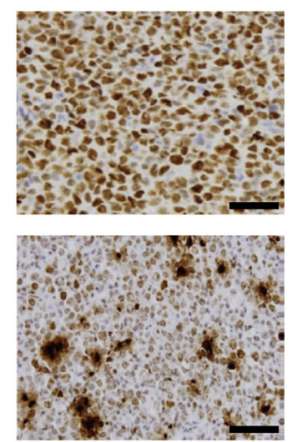

EDO $(80 \mathrm{mg} / \mathrm{kg})$

Figure 1 | Induction of global histone 3 (H3) hyperacetylation and increased DNA damage of tumor tissue after treatment with EDOS101. (a) Hyperacetylation of $\mathrm{H} 3$ and specific lysine residues in HL60 cells after treatment with EDO-S101 compared with bendamustine (Bend). (b) p53 and pH2AX staining in in vivo tumor tissue from Daudi Burkitt lymphoma samples taken at days 4 and 8 , showing greater DNA repair response after a single dose of 40 or $80 \mathrm{mg} / \mathrm{kg}$ EDO-S101 compared with vehicle. DMSO, dimethylsulfoxide. Bar $=100 \mu \mathrm{m}$. To optimize viewing of this image, please see the online version of this article at www.kidney-international.org. 


\section{Mouse}
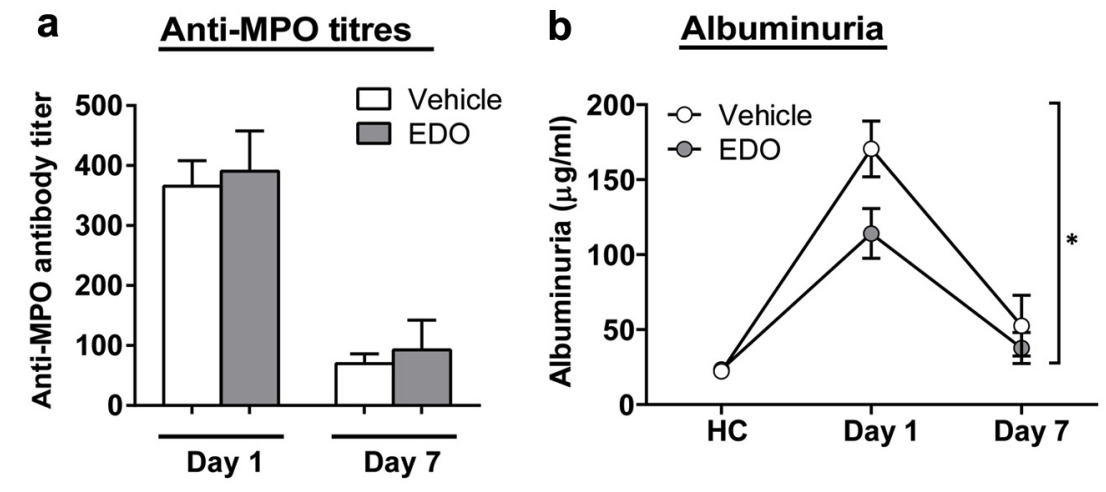

C $\quad$ Crescents
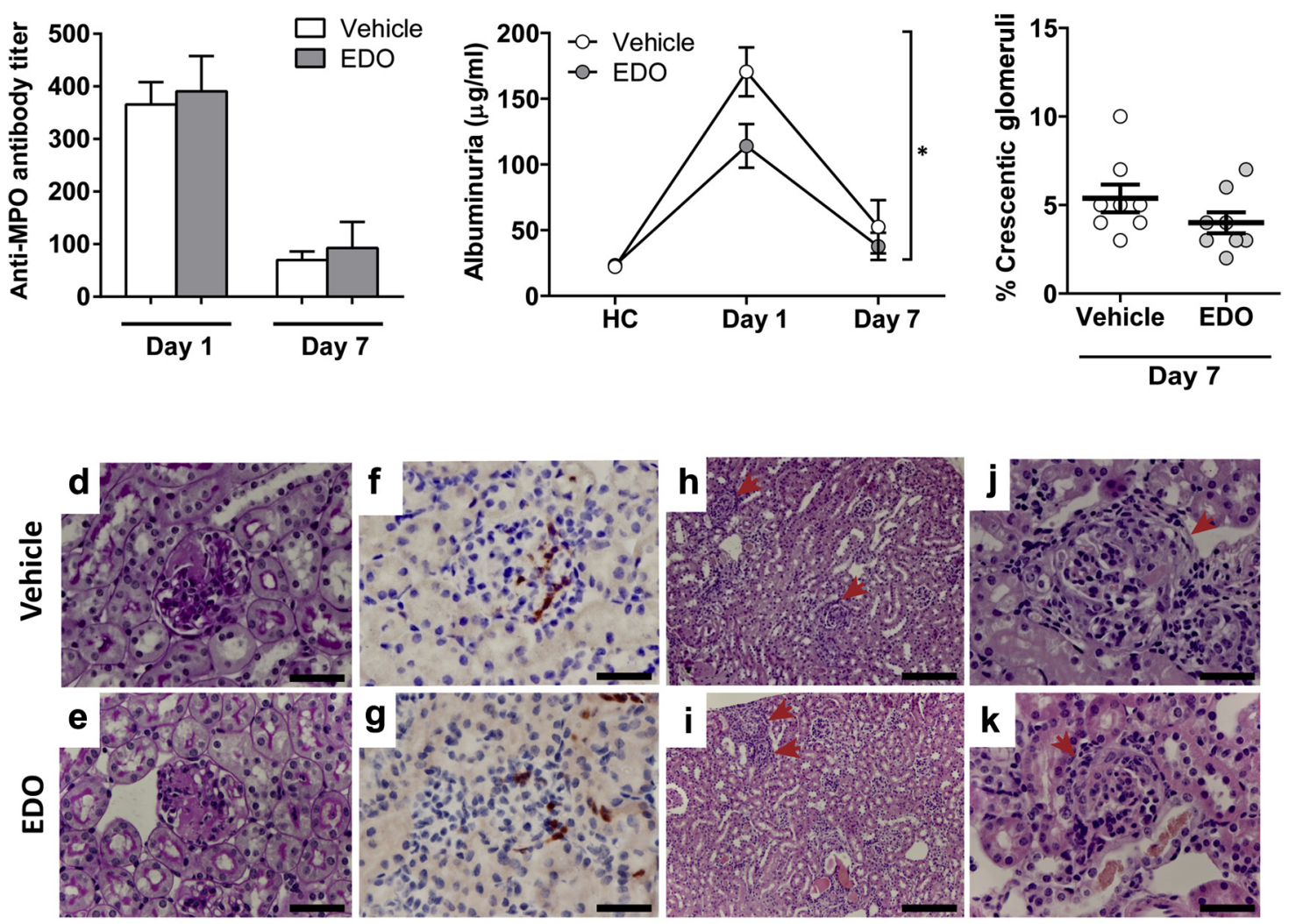

Figure 2 | Pretreatment with EDO-S101 in mice modestly decreases albuminuria but has no effect on glomerular histopathology. (a) Anti-myeloperoxidase (MPO) antibody titers after induction of anti-MPO lgG/lipopolysaccharide (LPS)-mediated glomerulonephritis in mice at days 1 and 7. (b) Albuminuria was significantly reduced in EDO-S101-pretreated mice compared with vehicle-treated controls. (c) Quantification of crescentic glomeruli showed no significant differences between treatment groups. (d-k) Representative glomerular histology in vehicle- and EDO-S101-pretreated mice. (d,e) Necrotic glomerulus (periodic acid-Schiff stain) on day 1 after disease induction in vehicle- and EDO-S101pretreated mice, respectively. $(\mathbf{f}, \mathbf{g})$ Neutrophil staining (Ly6G) 1 day after disease induction in vehicle- and EDO-S101-pretreated mice,

respectively. (h,i) Overview of renal cortical tissue (hematoxylin and eosin [H\&E] stain) on day 7 after disease induction with a similar number of crescentic glomeruli in both vehicle- and EDO-S101-pretreated mice (red arrows). (j,k) Crescentic glomeruli (H\&E stain) on day 7 after disease induction in vehicle- and EDO-S101-pretreated mice. Data are mean $\pm \mathrm{SEM}$. ${ }^{*} P<0.05$. HC, healthy control. (d-g,j,k) Bar $=50 \mu \mathrm{m}$. (h,i) Bar $=$ $100 \mu \mathrm{m}$. To optimize viewing of this image, please see the online version of this article at www.kidney-international.org.

\section{Treatment of the active model of EAV with EDO-S101 abolishes crescentic GN and reduces lung hemorrhage} As there was little effect of EDO-S101 on a direct antibodyinduced model of vasculitis, we hypothesized that the compound would be more effective in a model that entrains both arms of the adaptive immune system. We therefore tested EDO-S101 in the EAV rat model, mimicking the clinical scenario by treating from a point when the disease was clinically evident 28 days after immunization with MPO. We observed a significant reduction in anti-hMPO titer between vehicle- and EDO-S101-treated rats at day 56 (Figure 3a) as well as reduction in both hematuria and albuminuria (Figure $3 \mathrm{~b}$ and c). EAV rats treated with EDO-S101 exhibited dramatically improved $\mathrm{GN}$, with a reduction in crescent fraction from $6.1 \% \pm 1.7 \%$ to $0.2 \% \pm 0.1 \%(P<0.005$; Figure $3 \mathrm{~d})$. EDO-S101 significantly reduced lung hemorrhage severity (Figure 4), and there was a significant correlation between anti-MPO titer and glomerular injury (Supplementary Figure S4A and B).

\section{EDO-S101 increases monocyte and neutrophil fractions in mice and neutrophil fractions in rats}

Having shown a beneficial effect of EDO-S101 in EAV, we went on to dissect the corresponding cellular correlates using flow cytometry. EDO-S101 induced moderate overall leukopenia (Supplementary Figure S5). In the murine model, there was an increase in both monocyte and neutrophil fractions by day 7 in EDO-S101-treated mice, indicating a sparing of this cell type relative to other leukocytes (Figures $5 \mathrm{a}$ and $\mathrm{c}$ ). The fraction of activated monocytes and neutrophils was unaffected by EDO-S101 (Supplementary Figure S6). A similar 
Rat
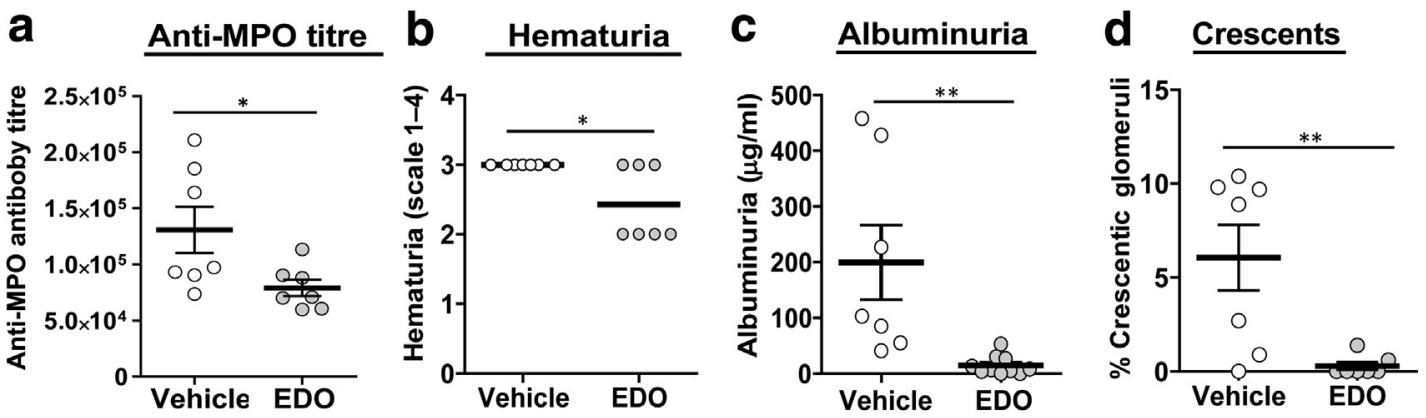

Day 56
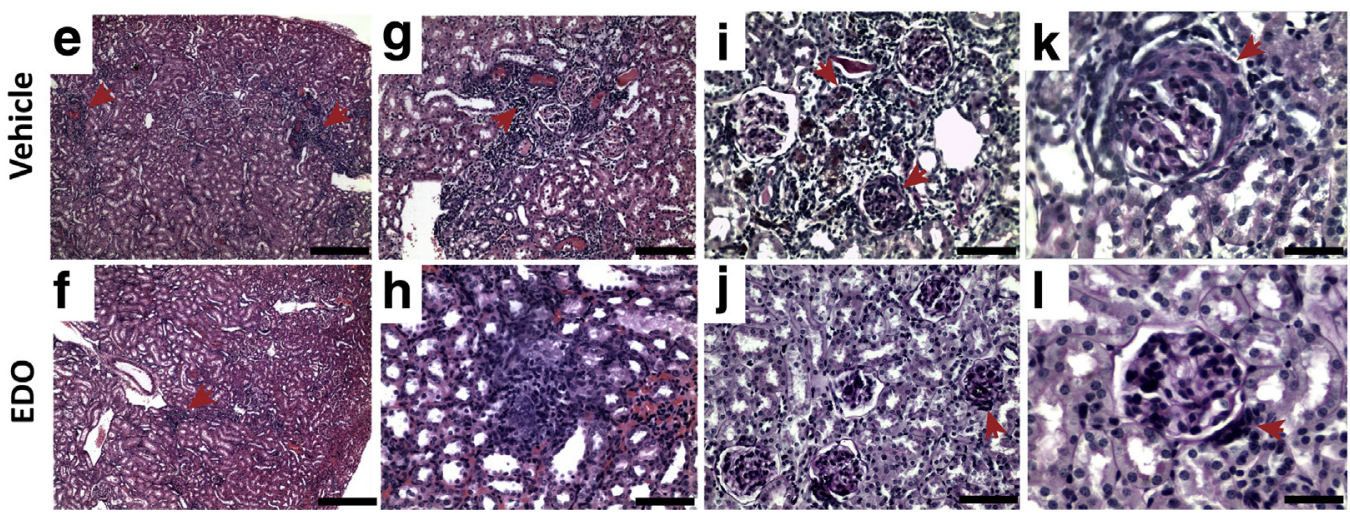

Figure 3 | EDO-S101 treatment in rats significantly decreases both hematuria and albuminuria and ameliorates proliferative and crescentic glomerulonephritis (GN). (a) Anti-human myeloperoxidase (MPO) antibody titers at day 56 after induction of experimental autoimmune vasculitis (EAV) in rats. Treatment with EDO-S101 in EAV rats led to significantly reduced (b) hematuria, (c) albuminuria, and (d) crescentic GN compared with vehicle-treated controls at day 56. (e-I) Representative glomerular histology in vehicle- and EDO-S101treated rats. (e) Foci of interstitial infiltrates and red cell tubular casts (hematoxylin and eosin [H\&E] stain; red arrows) in vehicle-treated rats and (f) mild interstitial infiltrates in EDO-S101-treated rats. (g) Mild GN, intense periglomerular inflammation, and red cell casts (H\&E stain; red arrows) in vehicle-treated rats. (h) Focus on inflammatory tubular destruction in EDO-S101-treated rats (H\&E stain). (i,k) Crescentic GN in vehicle-treated rats with intense periglomerular inflammation and red cell casts (periodic acid-Schiff [PAS] stain; red arrows). (j) Mild GN in EDOS101-treated rats with (I) focal glomerular necrosis and early crescent formation (PAS stain; red arrows). Data are mean \pm SEM. ${ }^{*} P<0.05$, ${ }^{*} \mathrm{P}<0.01$. (e,f) Bar $=500 \mu \mathrm{m}$. (g) Bar $=200 \mu \mathrm{m}$. (h-j) Bar $=100 \mu \mathrm{m}$. (k,l) Bar $=50 \mu \mathrm{m}$. To optimize viewing of this image, please see the online version of this article at www.kidney-international.org.

relative increase in neutrophil fraction (Figure 5d) was observed in EDO-S101-treated rats with EAV. The corresponding absolute neutrophil and monocyte counts are shown in Supplementary Figure S7.

\section{The dominant therapeutic cellular effect of EDO-S101 in EAV is on the lymphoid compartment}

Given the positive therapeutic effect of EDO-S101 in EAV rats, we included an analysis of natural killer (NK), B, and T cell populations to define the selective effect of EDO-S101. We observed a reduction in NK cell count during the evolution of EAV, which rebounded by day 56 (Supplementary Figure S8A). Although there was no significant effect on NK cell fraction after treatment with EDO-S101 (Figure 6a), a substantial reduction in rat $\mathrm{B}$ and $\mathrm{T}$ cells was observed (Figure $6 \mathrm{~b}$ and $\mathrm{c}$; Supplementary Figure S8B and C).
Treatment with EDO-S101 induces apoptosis in B cells and increases DNA damage in EAV tissue

To address its mechanistic effects on the immune system, we investigated the effects of EDO-S101 compared with those of bendamustine or vorinostat in a Seraphina Burkitt lymphoma cell line. EDO-S101 reduced B-cell viability (as determined by MTT [3-(4,5-dimethylthiazol-2-yl)-2,5-diphenyltetrazolium bromide] assay) and reduced proliferation (as determined by temporal cell count) (Figure. 7a and b). EDO-S101 increased B-cell subdiploid cell fractions (Figure 7c), cleaved poly(ADPribose) polymerase (PARP) levels (Figure $7 \mathrm{~d}$ ), and propidium iodide staining (Figure $7 \mathrm{e}-\mathrm{h}$ ). Further staining for p53 and $\mathrm{pH} 2 \mathrm{AX}$ in spleen tissue from rats treated with EDO-S101 showed a clear induction of both DNA damage markers (Figure 7i-k). Upregulation of p53 was particularly evident in EDO-S101-treated rats (Figure $7 \mathrm{~m}$ ), with extension beyond 


\section{Lung hemorrhage}

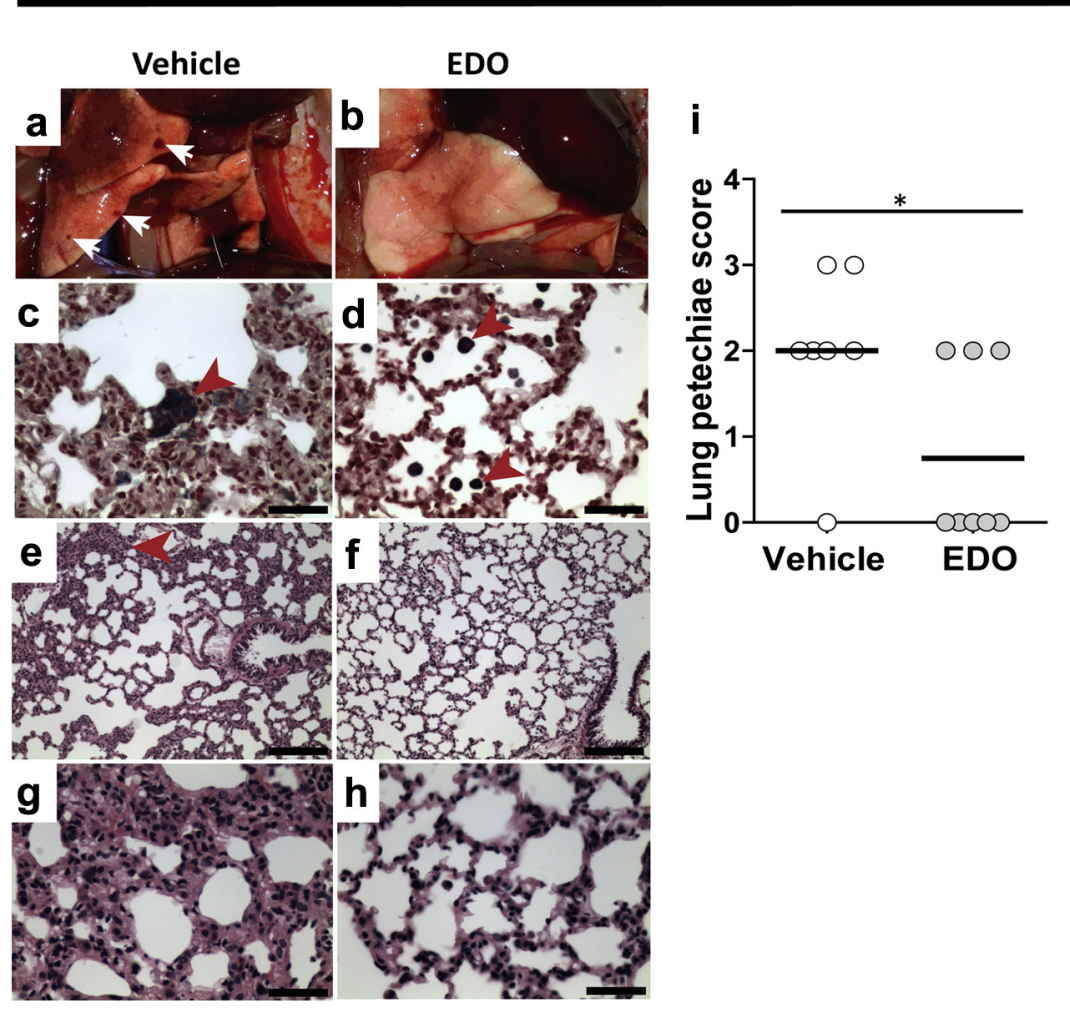

Figure 4 | Treatment with EDO-S101 significantly decreases lung hemorrhage in experimental autoimmune vasculitis rats. (a,b) The macroscopic lung petechiae score was determined by the assessment of the right lung of (a) vehicle-treated and (b) EDO-S101-treated animals. Lung hemorrhages are highlighted by white arrows. (c,d) Antemortem lung hemorrhage shown by the presence of Perls' Prussian blue-positive cells (blue; red arrow) in the lungs of (c) vehicle-treated and (d) EDO-S101-treated rats. (e,g) Alveolar hypocellularity of vehicletreated animals with thickening of alveolar walls (arrow). (f,h) EDO-S101-treated rats had minimal alveolar wall thickening (hematoxylin and eosin stain, original magnification $\times 100$ and $\times 400$ ). (i) Lung petechiae score of vehicle- and EDO-S101-treated rats. Data are mean \pm SEM. ${ }^{*} P<0.05$. (c, d, $\left.\mathbf{g}, \mathbf{h}\right) \mathrm{Bar}=50 \mu \mathrm{m}$. (e,f) Bar $=100 \mu \mathrm{m}$. To optimize viewing of this image, please see the online version of this article at www. kidney-international.org.

the restricted white pulp staining observed in vehicle-treated animals (Figure $7 \mathrm{j}$ ). Taken together, these results indicate an increase in apoptosis in EDO-S101-treated B cells and in EAV spleen tissue.

\section{DISCUSSION}

Existing alkylating agent therapy for AAV is largely effective at inducing remission, but at the cost of side effects and subsequent risk of relapse when the treatment is discontinued. We hypothesized that the effect of a low dose of the alkylating agent bendamustine could be potentiated by disrupting the chromatin structure, thereby making the DNA more accessible to the drug. This was achieved by creating a hybrid compound with the HDACi vorinostat and testing it in 2 wellestablished rodent models of MPO-AAV. Although the bifunctional molecule was relatively ineffective in a passive murine model, it proved highly efficacious in a rat model of AAV induced by active immunization with MPO, even when given after the establishment of disease. In a first experimental investigation of circulating leukocyte populations in the EAV model, we showed that this therapeutic effect was accompanied by a reduction in circulating $\mathrm{B}$ and $\mathrm{T}$ cells. This strongly suggests that the primary beneficial effect was mediated through action on the adaptive immune system.

It has been shown that pan-HDACis, such as vorinostat, increase histone 3 tail acetylation in transformed human cells. ${ }^{10}$ We previously confirmed this activity for EDO-S101 in MM1S cell lines, ${ }^{6}$ providing the first evidence that the compound indeed functions as a pan-HDACi. Additionally, in the present study, EDO-S101 induced hyperacetylation of the histone 3 tail at lysine residues $\mathrm{K} 9, \mathrm{~K} 14, \mathrm{~K} 23$, and $\mathrm{K} 56$ in extracts of HL60 cells and demonstrated greater acetylation capacity than did bendamustine alone.

Upon further investigation in EAV spleen tissue, we observed induction by EDO-S101 of pH2AX and particularly p53 (which extended well beyond the restricted white pulp staining observed in vehicle-treated animals), indicating increased DNA damage. On the one hand, EDO-S101 causes DNA injury via its alkylating function ( $\mathrm{pH} 2 \mathrm{AX}$ response), and on the other hand, it suppresses the homologous DNA repair response (p-ATR and p-ATM suppression). We hypothesize that this combined activity renders the compound more effective than a conventional alkylating agent, making it 


\section{Monocytes}
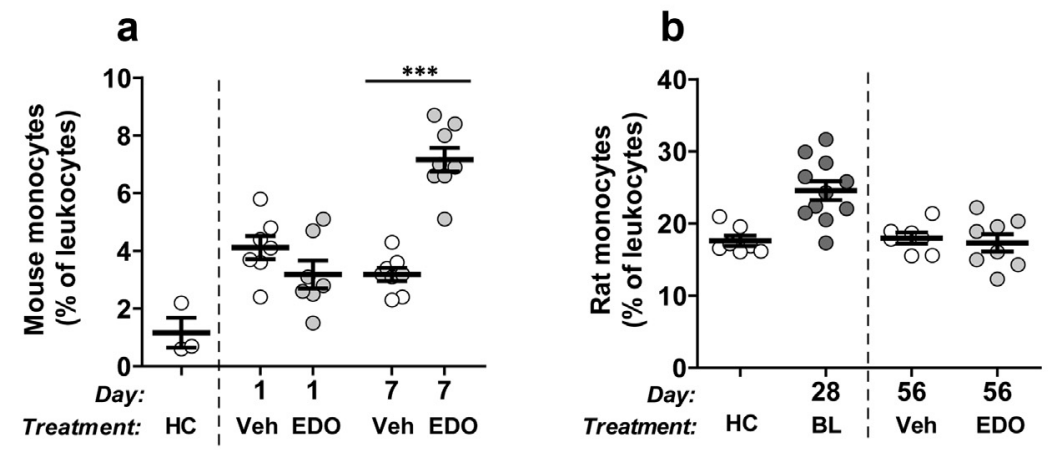

\section{Neutrophils}
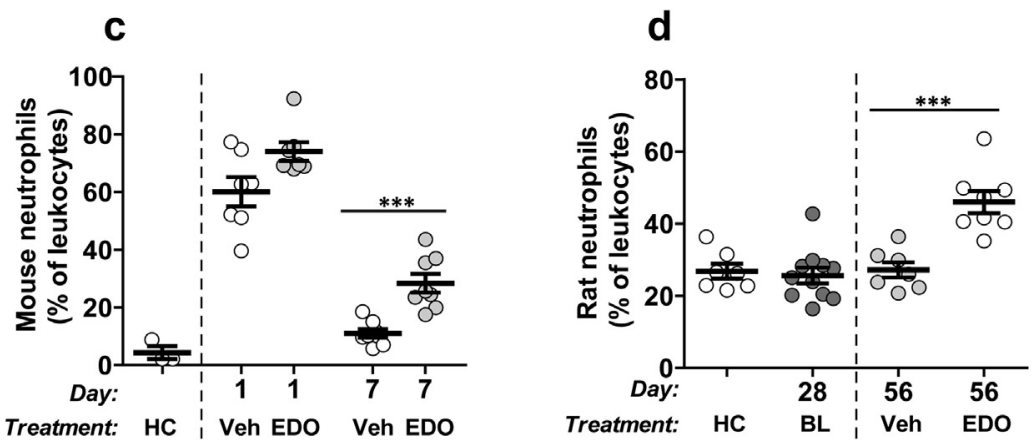

Figure 5 | Effect of treatment with EDO-S101 on relative myeloid cell fractions. (a) Pretreatment with EDO-S101 in mice significantly increased the percentage of monocytes from leukocytes at day 7. (b) In rats, treatment with EDO-S101 had no significant effect on the percentage of monocytes from leukocytes at day 56. (c) Pretreatment with EDO-S101 in mice significantly increased the percentage of neutrophils from leukocytes at day 7. (d) In rats, treatment with EDO-S101 increased the percentage of neutrophils from leukocytes compared with vehicle-treated controls. Data are mean $\pm \mathrm{SEM}$. ${ }^{* * *} \mathrm{P}<0.001$. BL, baseline disease in rats (day 28 postimmunization); $\mathrm{HC}$, unimmunized healthy control; Veh, vehicle.

applicable in various disease settings. ${ }^{6}$ These findings were complemented by proliferation assays in human B cells. We observed an increase in PARP cleavage in B cells treated with EDO-S101, which indicates cellular disassembly and thus an increase in apoptosis. Additionally, an increase in subdiploid DNA in EDO-S101-treated cells is also indicative of apoptosis. Consistent with the cell count and MTT assay data, EDO-S101 was significantly more potent at inducing apoptosis compared to equimolar concentrations of bendamustine and vorinostat, suggesting that it is possible to obtain similar effects with markedly reduced exposure to bendamustine.

To provide a mechanistic cellular basis for the activity of the drug, we performed a detailed assessment of peripheral leukocyte populations for the first time in these models. We observed a large reduction in peripheral leukocyte count on day 1 in the murine model, but a dramatic increase in the neutrophil component of these cells. This is consistent with prior work that shows a large increase in peripheral neutrophil count on day $1^{11}$ and is likely to be primarily a lipopolysaccharide (LPS) effect. As we did not include lymphocyte populations in the murine panel, we cannot determine the effect of LPS on these cell types. We suggest that the observed reduction in total leukocyte count in the murine model on day 1 is likely to be secondary to induction of acute lymphopenia, noting that lymphocytes are the dominant leukocyte type in mice. Prior work has demonstrated that the infusion of LPS causes acute lymphopenia, with up to $80 \%$ reduction in absolute lymphocyte count. ${ }^{12}$ It should also be noted that healthy control mice were studied at a different time than experimental mice, which may also have contributed to the marked difference.

Contrary to the effect of cyclophosphamide and other alkylating agents, EDO-S101 increased the fraction of circulating monocytes and neutrophils at the point of peak murine disease (day 7), and neutrophil fraction at peak EAV disease in the rat (day 56). Part of this increase in cell fractions is almost certainly due to relative reduction in other leukocyte types, with little change in absolute cell counts. The exception to this was the failure of the monocyte fraction to increase in EAV during EDO-S101 treatment, reflected in the reduction in absolute monocyte count in the rat. This difference in monocyte response between the mouse and the rat may be due to lingering effects of LPS in the mouse that were not evident with the longer EAV time course. Although the dominant effect of EDO-S101 appears to be on lymphocytes, this reduction in circulating rat monocyte numbers may have contributed to a reduction in glomerular recruitment and severity of GN. 


\section{Rat}

a

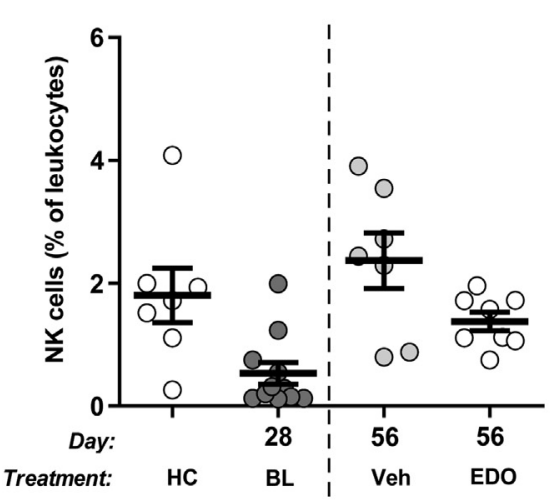

b

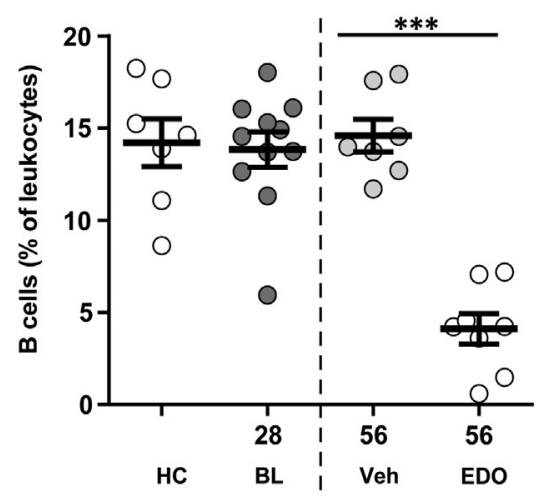

C

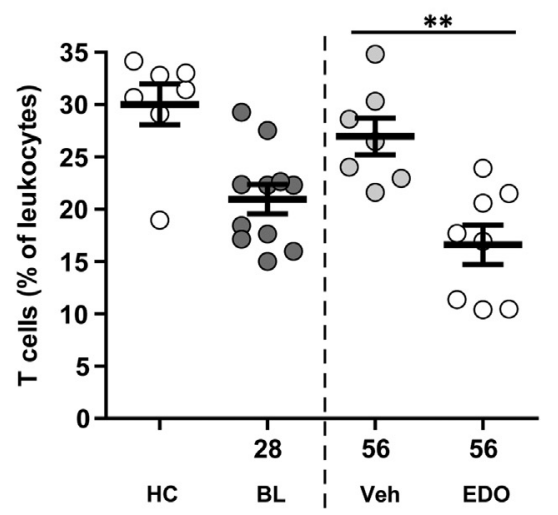

Figure 6| Treatment with EDO-S101 results in selective depletion of B and T cells in rats compared with vehicle-treated controls. (a) There was a nonsignificant trend toward a decrease in the percentage of natural killer (NK) cells from leukocytes in rats compared with vehicletreated controls. There was a significant decrease in (b) B-cell and (c) T-cell percentages from leukocytes after treatment with EDO-S101 in rats compared with vehicle-treated controls. Data are mean $\pm \mathrm{SEM}$. ${ }^{* *} P<0.01,{ }^{* * *} P<0.001$. BL, baseline disease in rats (day 28 postimmunization); $\mathrm{HC}$, unimmunized healthy control; Veh, vehicle.

EDO-S101 reduced the absolute monocyte cell count (but not fraction) on day 1 in the mouse, without significantly affecting the neutrophil count. Overall, neutrophils were relatively spared compared to other leukocyte populations in both rodent models, with a significant increase in circulating fraction by day 7 in the mouse and day 56 in the rat. Prior work in the murine model has demonstrated the importance of neutrophils in this condition. ${ }^{13}$ This relative sparing of neutrophils may partially explain why the compound was ineffective in this neutrophil-dependent murine model. Although we did not measure this directly, in addition to its effect on the leukocyte count, the HDACi effect may have altered the neutrophil chromatin structure. Ciavatta and coworkers have proposed epigenetic disruption of proteinase 3 and MPO gene silencing as a potential mechanism of triggering autoimmunity in patients with $\mathrm{AAV} .^{14,15}$ Although it is unlikely that these epigenetic effects are at play in these rodent models, it is interesting to speculate that EDO-S101 may target similar epigenetic effects, representing an additional potential mechanism of action.

Previous clinical and preclinical studies have shown that EDO-S101 exerts potent therapeutic effects against hematological malignancies and works on the adaptive immune response. ${ }^{7,8,16-18}$ Given its ability to suppress $\mathrm{B}$ and $\mathrm{T}$ cells effectively in preclinical studies, it seemed plausible that treatment with EDO-S101 may provide therapeutic effects in autoimmune disease, such as AAV. Indeed, in this study, we observed a striking depletion of $\mathrm{B}$ and $\mathrm{T}$ cells in the EAV model after EDO-S101 treatment as well as a significant reduction in anti-hMPO antibodies in the EAV model.

When considering limitations in this study, it important to note experimental differences between the rat and mouse models. For example, no absolute monocyte or neutrophil cell counts were observed in mice; these were calculated from total leukocyte counts. Additionally, we did not include analysis of lymphocyte populations (B, T, and NK cells) in the murine model as performed in the rat study, although little therapeutic effect was observed in mice. Furthermore, healthy control mice were studied separately from experimental mice. Neither of these murine or rat models are satisfactory models of human ANCA vasculitis, not least because of the relatively mild disease phenotype in both. Nevertheless, it is worth highlighting that they reflect different aspects of AAV pathogenesis, thereby allowing us to study the effects of EDOS101 on the innate and adaptive immune responses. Another interesting aspect to consider would be further phenotyping of the p53/pH2AX-positive spleen cells to determine whether these are of $\mathrm{B}$ or T-cell origin (e.g., via CD3 or CD19 staining). Our findings do not definitively exclude the possibility that the therapeutic effects simply occurred as a nonspecific consequence of leukopenia. Vorinostat, being a pan-HDACi, would have wide-ranging effects on many cell types. However, the clear dichotomy between the effects of EDO-S101 on the innate and adaptive immune responses argues against this; a nonspecific effect would be expected to affect most on the rapidly renewing neutrophil pool.

To conclude, we have demonstrated varying therapeutic effects of a novel alkylating HDACi fusion molecule, EDOS101, in 2 contrasting models of MPO-ANCA vasculitis. Our data indicate a selective effect of EDO-S101 on the adaptive immune response resulting in the amelioration of GN and lung hemorrhage in the EAV model. Given the induction of apoptosis in proliferating human $\mathrm{B}$ cells and the rat $\mathrm{B}$ cell depletion in vivo, we speculate that EDO-S101 may act on the autoreactive B-cell pool. Further work is required to determine optimal dosing strategy, whether an orally active version can be developed, and to assess whether the 


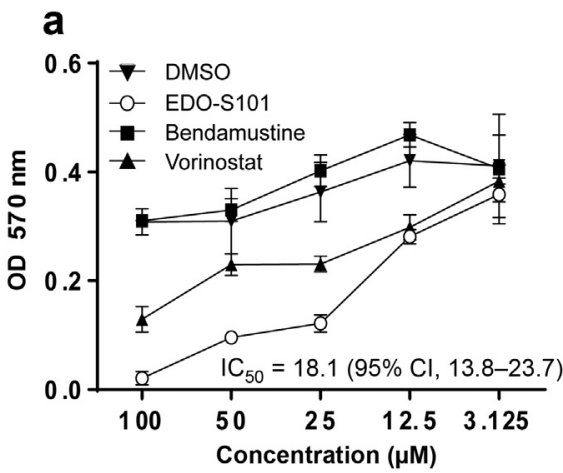

C

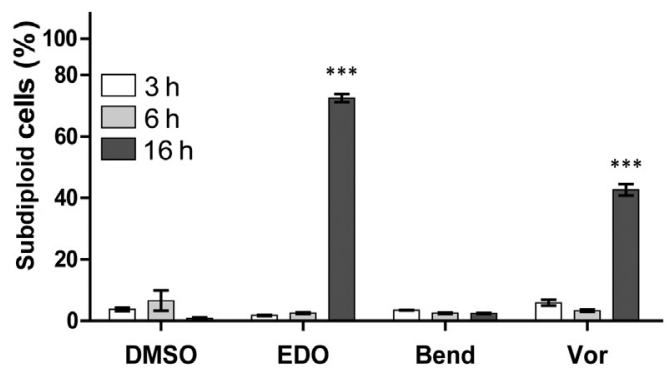

e

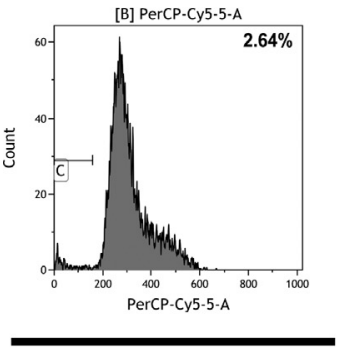

DMSO f

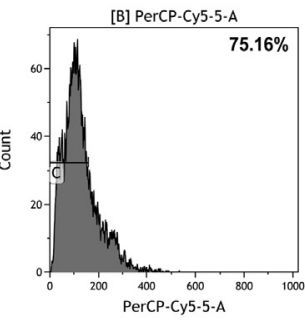

EDO

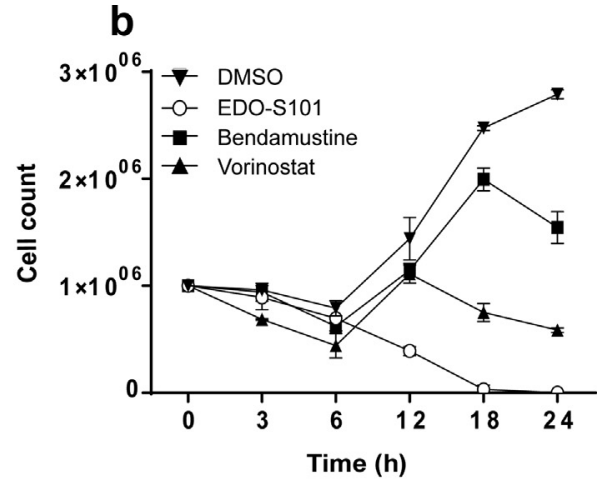

d
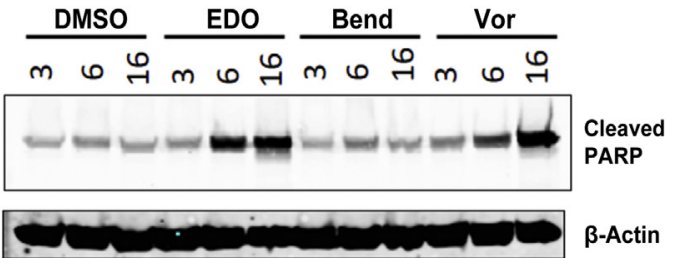

g

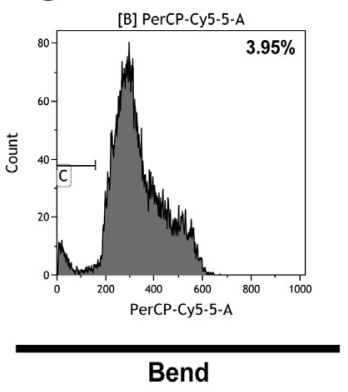

\section{k}

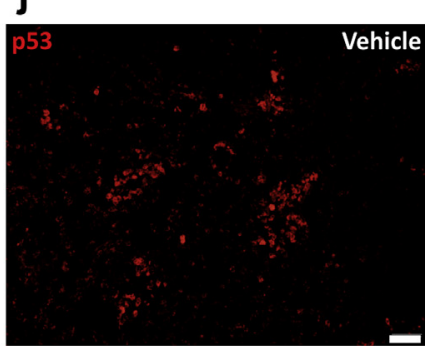

m

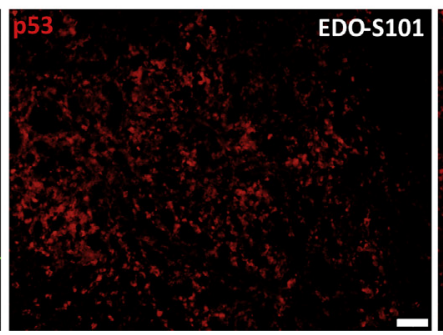

n h

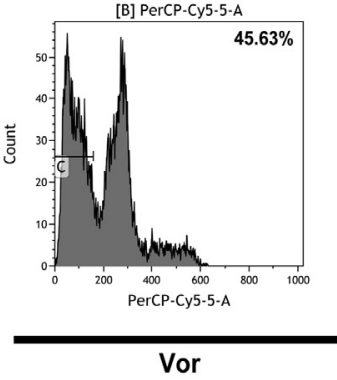

Vor
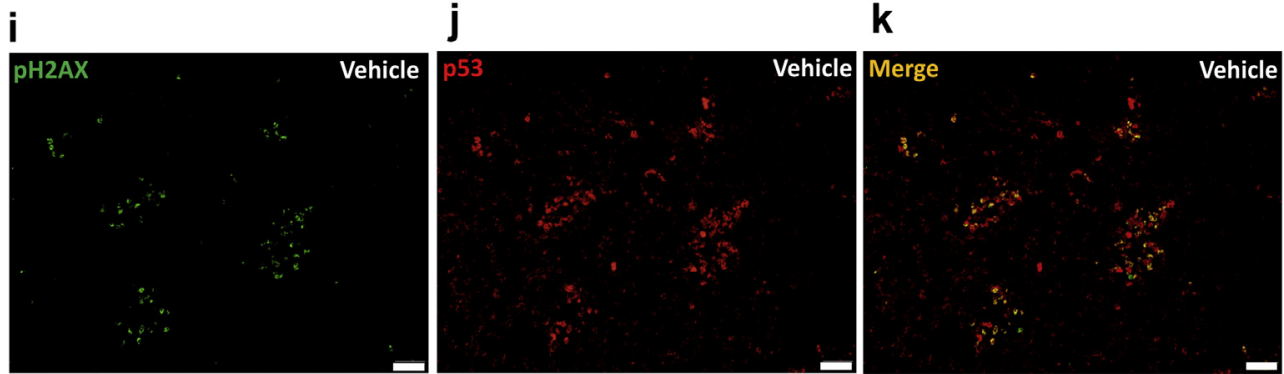

I
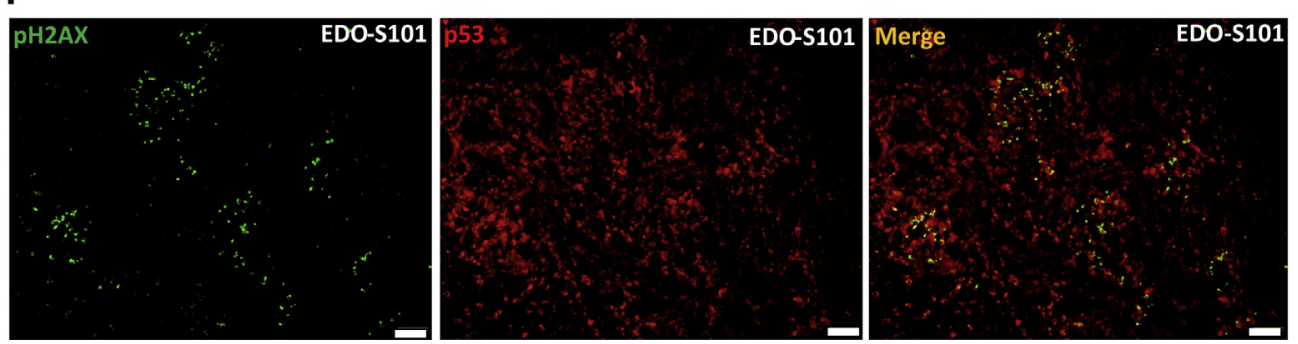

Figure 7| Treatment with EDO-S101 induces apoptosis in a B cell line and increases DNA damage in experimental autoimmune vasculitis tissue. (a) Seraphina BL cells were cultured at $1 \times 10^{6}$ cells $/ \mathrm{ml}$ in the presence of increasing concentrations of EDO-S101, bendamustine (Bend), or vorinostat (Vor), and viability of cells was assessed by MTT assay 16 hours later. Viability assessed at 6 hours (continued) 
bifunctional molecule can reduce infectious complications. The present data suggest that EDO-S101 is a promising novel agent for the treatment of AAV and support the pursuit of these additional preclinical studies.

\section{METHODS}

\section{Animals}

$\mathrm{MPO}^{-} /^{-}$mice $\left(\mathrm{B} 6.129 \mathrm{X} 1-\mathrm{MPO}^{\text {tmlLus }} / \mathrm{J}\right)$ were purchased from the Jackson Laboratory (Bar Harbor, ME) and bred in-house. Female C57BL/6J mice (8-10 weeks old) were purchased from Harlan Laboratories B.V. (Horst, The Netherlands). Wistar Kyoto rats were derived from the inbred colony of authors CDP and FWKT and bred by Charles River UK Ltd. (Margate, UK). Equal numbers of male and female rats ( 6 weeks old) were used throughout the experiment in both treatment groups. A mouse xenograft model was used for investigating the DNA-damaging effects of EDO-S101 in tumor tissue as previously described. ${ }^{6}$ All experiments were approved by the local ethics committees and performed according to the guidelines on the protection of animals used for scientific purposes at the University Medical Centre Groningen, Trinity College Dublin, and Mundipharma-EDO GmbH (European Union Directive 2010/63/ EU) (see Supplementary Materials and Methods for further details).

\section{Western blotting}

Cells were centrifuged at $10,000 \mathrm{~g}$ for 2 minutes, washed with phosphate-buffered saline, and lysed in ice-cold lysis buffer $(140 \mathrm{mM}$ $\mathrm{NaCl}, 10 \mathrm{mM}$ ethylenediamine tetraacetic acid, $10 \%$ glycerol, $1 \%$ Nonidet P-40, $20 \mathrm{mM}$ tris(hydroxymethyl)-aminomethane [pH 7.0], $1 \mu \mathrm{M}$ pepstatin, $1 \mu \mathrm{g} / \mathrm{ml}$ aprotinin, $1 \mu \mathrm{g} / \mathrm{ml}$ leupeptin, and $1 \mathrm{mM}$ sodium orthovanadate). Western blots were probed with antibodies to p-ATR, p-ATM, checkpoint kinase 2, cleaved PARP Asp214 (Cell Signaling Technology, Boston, MA), and $\beta$-Actin (Sigma-Aldrich, Ireland).

\section{Murine passive transfer vasculitis disease induction and treatment protocol}

Male and female C57Bl/6J mice were randomly divided into 4 groups: vehicle pretreated and killed at day 1 or 7 ( $n=8$ per group) or EDO-S101 pretreated and killed at day 1 or 7 ( $n=8$ per group). Mice received EDO-S101 (25 mg/kg) or vehicle i.v. 1 day before disease induction. On the following day, disease was induced via i.v. administration of $1.5 \mathrm{mg}$ of anti-MPO IgG, followed by LPS administration i.p. $(5 \mu \mathrm{g} / \mathrm{g}) 1$ hour later (Escherichia coli serotype O26:B6, Sigma-Aldrich) (see Supplementary Materials and Methods for further details on polyclonal mouse anti-MPO IgG production).

\section{Urine and plasma analysis of murine samples}

Urine samples were collected from mice at varying time points using metabolic cages. Hematuria was assessed using the dipstick method (score 0-4) at days 1 to 7 using Combur-Test strips (Roche
Diagnostics BV, Almere, The Netherlands). Albuminuria (days 1 and 7) was measured by enzyme-linked immunosorbent assay (ELISA) (quantitation kit, Bethyl Laboratories, TX) following the manufacturer's instructions. Plasma samples were tested for circulating antiMPO antibody titers by ELISA at days 1 and 7 as described above using a serum pool from MPO-immunized $\mathrm{MPO}^{-1-}$ mice for reference as previously described. ${ }^{19}$

\section{Flow cytometry of murine samples}

Automated total and differential white blood cell counts were determined at the time of killing (days 1 and 7) by using the XT1800iV (Sysmex, Etten-Leur, The Netherlands). Neutrophils $\left(\right.$ Ly6G $\left.{ }^{+} \mathrm{CD} 11 \mathrm{~b}^{\text {high }}\right)$ and monocytes $\left(\mathrm{Ly} 6 \mathrm{G}^{-} \mathrm{CD} 11 \mathrm{~b}^{+}\right)$as well as their activation status were analyzed in peripheral blood by flow cytometry with an FACSCalibur flow cytometer (BD Biosciences, UK) (see Supplementary Materials and Methods for further details).

\section{EAV protocol}

EAV, a model of MPO-ANCA vasculitis, was induced in male and female rats as previously described. ${ }^{20}$ Unimmunized animals were included to provide a comparison to the basal state. Co-housed rats were randomly divided into 2 groups: vehicle or EDO-S101 (10 mg/ $\mathrm{kg}$ ) treated ( $\mathrm{n}=7-8$ per group) and received treatment i.v. into the lateral tail vein beginning at day 28 (baseline), given that we have previously shown well-established disease at day 28 in this model. ${ }^{21}$ All rats were killed at day 56 (see Supplementary Materials and Methods for further details).

\section{Urine and plasma analysis of rat samples}

Urine samples were collected from rats at day 56 using metabolic cages, and hematuria was assessed using the dipstick method (score 0-4) using Multistix 10 SG reagent strips (Siemens, Dublin, Ireland). Albuminuria was measured by ELISA (Nephrat kit, Exocell, The Netherlands) following the manufacturer's instructions. Plasma samples were tested for circulating anti-hMPO antibody titers by ELISA at day 56 as previously described ${ }^{19,20}$ (see Supplementary Materials and Methods for further details).

\section{Flow cytometry of rat samples}

Peripheral blood was taken from healthy control rats on day 28 postimmunization (baseline disease) and at the time of killing (day 56) and analyzed by flow cytometry to determine leukocyte subsets. Samples were analyzed with a CyAn ADP analyzer (Beckman Coulter Life Sciences, CA). Subsequent analysis was performed using Kaluza analysis software version 1.2 (Beckman Coulter Life Sciences). Frequencies and absolute numbers of the following leukocyte subsets were determined: total leukocytes $\left(\mathrm{CD} 45^{+}\right)$, neutrophils (SSC ${ }^{\text {hi }} \mathrm{RP}-$ $\left.1^{+}\right)$, monocytes $\left(\mathrm{CD}^{-} \mathrm{CD}^{+}\right), \mathrm{B}$ cells $\left(\mathrm{CD} 4 \mathrm{RA}^{+}\right)$, T cells $\left(\mathrm{CD} 3^{+}\right)$, and NK cells $\left(\mathrm{CD}^{-} \mathrm{CD}^{-} 161 \mathrm{a}^{+}\right.$) (see Supplementary Materials and Methods for further details).

Figure 7 | (continued) is shown in Supplementary Figure S9. (b) BL cells were cultured in $25 \mu \mathrm{M}$ EDO-S101, Bend, or Vor, and cells were counted at indicated time points. (c) The percentage of subdiploid cells was measured from the DNA content of the treated cells. (d) BL cells were cultured in $25 \mu \mathrm{M}$ EDO-S101, Bend, or Vor and harvested at the indicated time points and then probed for levels of cleaved poly(ADP-ribose) polymerase (PARP; Asp214). (e-h) Representative histograms of 16-hour treated cells, fixed and stained with propidium iodide for DNA content analysis. Representative photomicrographs of spleen tissue from (i-k) vehicle- and (I-n) EDO-S101-treated rats stained for p53 and $\mathrm{pH} 2 \mathrm{AX}$ are indicated. There is a clear induction of both markers in EDO-S101-treated rats, particularly (m) p53, which extends beyond the restricted white pulp staining observed in (j) vehicle-treated animals. Error bars indicate mean $\pm \mathrm{SEM}$; ${ }^{* *} P<0.001$. $\mathrm{BL}$, baseline; $\mathrm{Cl}$, confidence interval; DMSO, dimethylsulfoxide; $\mathrm{IC}_{50}$, half-maximal inhibitory concentration; OD, optical density. (i-n) Bar $=50 \mu \mathrm{m}$. To optimize viewing of this image, please see the online version of this article at www.kidney-international.org. 


\section{Cell viability and analysis of DNA content in human B cells}

Seraphina Burkitt lymphoma cells were treated as described in figure legends with dimethylsulfoxide control, EDO-S101, bendamustine (Sigma-Aldrich), or vorinostat (Sigma-Aldrich). After the indicated times, cells were harvested and counted. Cell viability was analyzed by MTT assay. Additionally, propidium iodide-stained cells were analyzed for DNA content by flow cytometry with a CyAn ADP analyzer (Beckman Coulter Life Sciences) (see Supplementary Materials and Methods for further details).

\section{Immunohistopathological analysis}

Activation of p53 induces the expression of various gene products, which in turn can either prevent proliferation of damaged cells or induce apoptosis, thereby removing damaged cells from the body. ${ }^{22}$ Phosphorylation of H2AX plays a major role in the DNA damage response and is required for the assembly of DNA repair proteins. ${ }^{23}$ Therefore, we investigated these molecules, given their role in apoptosis and DNA damage. ${ }^{9,23}$ For analysis of the xenograft model, selected tumors excised from the treated and control mice were stained for anti-pH2AX and anti-p53 (Cell Signaling Technology). To further investigate the effect of EDO-S101 on DNA damage, rat spleen sections $(10 \mu \mathrm{m})$ were also stained for anti-pH2AX and antip53 (Cell Signaling Technology). For analysis of the vasculitis model, kidney sections $(4 \mu \mathrm{m})$ were stained with periodic acid-Schiff and hematoxylin and eosin stains to assess morphology as previously described. ${ }^{19-21,24}$ In addition to renal pathology, rats with EAV develop pulmonary vasculitis and hemorrhage as previously described $^{25}$ (see Supplementary Methods for further details).

\section{Statistical analysis}

All statistical analyses were performed using Prism 6.0 software (GraphPad Software Inc., La Jolla, CA). Mouse hematuria and albuminuria were analyzed using 2-way analysis of variance, including time as a variable. All other comparisons between 2 groups were evaluated using the nonparametric Mann-Whitney $U$ test. Differences were considered statistically significant when $P<0.05$. Data are expressed as mean \pm SEM values per experimental group.

\section{DISCLOSURE}

TM is an employee of Mundipharma-EDO GmbH. FWKT has received research project grants from AstraZeneca, Baxter Biosciences, Boehringer Ingelheim, and Medlmmune and has consultancy agreements with Rigel Pharmaceuticals, Novartis, and Baxter Biosciences. CDP has received a research project grant from GlaxoSmithKline. The experimental work was funded by Mundipharma-EDO GmbH. All the other authors declared no competing interests.

\section{ACKNOWLEDGMENTS}

This work was supported by Science Foundation Ireland grant $11 / \mathrm{Y}$ / B2093. FWKT is supported by the Diamond Fund from Imperial College Healthcare Charity and Ken and Mary Minton Chair of Renal Medicine.

\section{AUTHOR CONTRIBUTIONS}

DD participated in collection and/or assembly of data, data analysis/ interpretation, and manuscript writing. MMvT, VOR, and ECOB participated in the study design and data analysis/interpretation. $G B$, $\mathrm{BF}, \mathrm{FBH}, \mathrm{EL}, \mathrm{CDP}$, and FWKT participated in the data analysis/ interpretation and manuscript writing. TM, PH, and MAL participated in the study design, conception, and coordination and helped draft the final manuscript. All authors read and approved the final manuscript.

\section{SUPPLEMENTARY MATERIAL}

\section{Supplementary Materials and Methods.}

Figure S1. DNA repair response in tumor samples after treatment with EDO-S101. DNA repair response taken from Daudi Burkitt lymphoma samples at days 4 and 8 after dosing. p-ATR and p-ATM are dose dependently suppressed by EDO-S101, while p-CHK2 is strongly upregulated at day 4 but returns to undetectable levels at day 8 . Figure S2. Pretreatment with EDO-S101 in mice has no effect on hematuria. (A) Urinalysis and anti-myeloperoxidase (MPO) antibody titration curves after induction of anti-MPO lgG/LPS-mediated glomerulonephritis in mice at days 1 and 7. (B) Hematuria score in vehicle- and EDO-S101-pretreated mice was highest at day 1 and gradually decreased in time, with no significant effect of EDO-S101 pretreatment. (C) Anti-hMPO antibody titration curves after induction of experimental autoimmune vasculitis in rats at day 56. Data are mean \pm SEM.

Figure S3. EDO-S101 induces a significant correlation between antimyeloperoxidase (MPO) titer and urine/histological abnormalities in the rat model. Correlation analysis showed a significant correlation between anti-MPO titer and (A) glomerular injury and (B) albuminuria $(r>0.7)$ in the EDO-S101-treated group.

Figure S4. Pretreatment with EDO-S101 in mice has no effect on glomerular histopathology, and EDO-S101 treatment in rats has no effect on proliferative glomerulonephritis $(\mathrm{GN})$ or tubulointerstitial nephritis (TIN) score. (A) Quantification of glomerular neutrophils (Ly6G) 1 day after disease induction in mice showed no significant differences between treatment groups. (B) Quantification of necrotic or abnormal glomeruli (periodic acid-Schiff stain) 1 day after disease induction in mice showed no significant differences between treatment groups. (C) Quantification of proliferative GN (proportion of glomeruli classified as abnormal) was nonsignificantly decreased in EDO-S101-treated rats compared with vehicle-treated controls. (D) There was no significant difference in TIN score between EDO-S101and vehicle-treated rats. Data are mean \pm SEM.

Figure S5. Pretreatment and treatment with EDO-S101 induced leukopenia in both mouse and rat models. (A) At days 1 and 7, total leukocyte counts were significantly lower in mice that received EDOS101 than in vehicle-treated controls. (B) At day 56, treatment with EDO-S101 significantly reduced leukocyte counts in rats compared with vehicle-treated controls, suggesting that EDO-S101 induces leukopenia in both models. The normal leukocyte range is indicated in healthy controls ( $\mathrm{HCS}$ ) in both mice and rats. Baseline (BL) in rats denotes baseline disease before treatment with EDO-S101 (day 28 postimmunization). Data are mean \pm SEM. ${ }^{*} P<0.05$, ${ }^{* *} P<0.01$, ***P $<0.001$.

Figure S6. Effect of treatment with EDO-S101 on mouse myeloid cell activation. There was no significant effect of EDO-S101 pretreatment on the percentage of activated (A) neutrophils or (B) monocytes compared with vehicle (Veh) pretreatment on day 1 or day 7. Data are mean \pm SEM.

Figure S7. Treatment with EDO-S101 decreases the number of circulating monocytes in both mice and rats. (A) At day 1, pretreatment with EDO-S101 in mice significantly reduced the circulating monocyte count compared with vehicle-treated controls. (B) In rats, treatment with EDO-S101 significantly reduced the circulating monocyte count compared with vehicle-treated controls at day 56 . There was no significant effect of EDO-S101 treatment on circulating neutrophil counts in (C) mice or (D) rats. $\mathrm{HC}$ represents unimmunized healthy controls, while $\mathrm{BL}$ denotes baseline disease in rats (day 28 postimmunization). Data are mean \pm SEM. ${ }^{*} P<0.05$, ${ }^{* *} P<0.01$, $* * * P<0.001$.

Figure S8. Treatment with EDO-S101 results in selective depletion of natural killer (NK) cells, B cells, and T cells in rats compared with vehicle-treated controls. (A) Circulating NK cells were elevated in rats 
that received vehicle as compared with baseline levels; however, treatment with EDO-S101 blocked this increase and resulted in a significant decrease in NK cells. There was also a significant decrease in (B) B cells and (C) T cells after treatment with EDO-S101 compared with vehicle-treated controls. $\mathrm{HC}$ represents unimmunized healthy controls, while BL denotes baseline disease in rats (day 28 postimmunization). Data are mean \pm SEM. ${ }^{* *} P<0.01,{ }^{* * *} P<0.001$. Figure S9. DNA content analysis in B cells after 6-hour treatment with EDO-S101. (A-D) BL cells were cultured in $25 \mu \mathrm{M}$ EDO-S101, bendamustine (Bend), or vorinostat (Vor) for 6 hours and then fixed and stained with propidium iodide for DNA content analysis. Supplementary material is linked to the online version of the paper at www.kidney-international.org.

\section{REFERENCES}

1. Kallenberg CG. Pathogenesis of ANCA-associated vasculitides. Ann Rheum Dis. 2011;70(suppl 1):i59-i63.

2. Jennette JC, Falk RJ. Pathogenesis of antineutrophil cytoplasmic autoantibody-mediated disease. Nat Rev Rheumatol. 2014;10:463-473.

3. Tesar V, Hruskova Z. Limitations of standard immunosuppressive treatment in ANCA-associated vasculitis and lupus nephritis. Nephron Clin Pract. 2014;128:205-215.

4. Frew AJ, Johnstone RW, Bolden JE. Enhancing the apoptotic and therapeutic effects of HDAC inhibitors. Cancer Lett. 2009;280:125-133.

5. Hancock WW, Akimova T, Beier UH, et al. HDAC inhibitor therapy in autoimmunity and transplantation. Ann Rheum Dis. 2012;71(suppl 2):i46-i54.

6. Mehrling T, Chen Y. The alkylating-HDAC inhibition fusion principle: taking chemotherapy to the next level with the first in class molecule EDO-S101. Anticancer Agents Med Chem. 2016;16:20-28.

7. López-Iglesias AA, San-Segundo L, González-Méndez L, et al. The alkylating histone deacetylase inhibitor fusion molecule Edo-S101 displays full bi-functional properties in preclinical models of hematological malignancies. Blood. 2014;124:2100.

8. Kraus M, Bader J, Mehrling T, Driessen C. Edo-S101, a new alkylating histone-deacetylase inhibitor (HDACi) fusion molecule, has superior activity against myeloma and B cell lymphoma and strong synergy with proteasome inhibitors in vitro. Blood. 2014;124:2249.

9. Di Benedetto A, Ercolani C, Mottolese M, et al. Analysis of the ATR-Chk1 and ATM-Chk2 pathways in male breast cancer revealed the prognostic significance of ATR expression. Sci Rep. 2017;7:8078.

10. Drogaris $P$, Villeneuve $V$, Pomiès $C$, et al. Histone deacetylase inhibitors globally enhance $\mathrm{H} 3 / \mathrm{H} 4$ tail acetylation without affecting H3 lysine 56 acetylation. Sci Rep. 2012;2:220.
11. Wang $\mathrm{Q}$, van Timmeren MM, Petersen $\mathrm{AH}$, et al. Age-determined severity of anti-myeloperoxidase autoantibody-mediated glomerulonephritis in mice. Nephrol Dial Transplant. 2017;32:254-264.

12. Copeland S, Warren HS, Lowry SF, et al. Acute inflammatory response to endotoxin in mice and humans. Clin Diag Lab Immunol. 2005;12:60-67.

13. Xiao H, Heeringa $\mathrm{P}$, Liu Z, et al. The role of neutrophils in the induction of glomerulonephritis by anti-myeloperoxidase antibodies. Am J Pathol. 2005;167:39-45.

14. Ciavatta DJ, Yang J, Preston GA, et al. Epigenetic basis for aberrant upregulation of autoantigen genes in humans with ANCA vasculitis. J Clin Invest. 2010;120:3209-3219.

15. McInnis EA, Badhwar AK, Muthigi A, et al. Dysregulation of autoantigen genes in ANCA-associated vasculitis involves alternative transcripts and new protein synthesis. J Am Soc Nephrol. 2015;26:390-399.

16. Besse $\mathrm{L}$, Kraus M, Besse A, et al. The first in class, alkylator-histonedeacetylase-inhibitor fusion molecule Edo-S101 in combination with proteasome inhibitors induces highly synergistic pro-apoptotic signaling through UPR activation and suppression of c-Myc and BCL2 in multiple myeloma. Blood. 2016;128:4466.

17. De Filippi R, Crisci S, Cillo M, et al. The first-in-class alkylating histonedeacetylase inhibitor (HDACi) fusion molecule Edo-S101 exerts potent preclinical activity against tumor cells of Hodgkin lymphoma (HL) including bendamustine-resistant clones. Blood. 2015;126:2481.

18. López-Iglesias AA, Herrero AB, San-Segundo $L$, et al. The hybrid molecule, Edo-S101, impairs double strand breaks repair in multiple myeloma and synergizes with bortezomib and dexamethasone. Blood. 2015;126:5354.

19. Little MA, Bhangal $G$, Smyth $C L$, et al. Therapeutic effect of anti-TNF- $\alpha$ antibodies in an experimental model of anti-neutrophil cytoplasm antibody-associated systemic vasculitis. J Am Soc Nephrol. 2006;17: 160-169.

20. Little MA, Smyth L, Salama AD, et al. Experimental autoimmune vasculitis: an animal model of anti-neutrophil cytoplasmic autoantibodyassociated systemic vasculitis. Am J Pathol. 2009;174:1212-1220.

21. O'Reilly VP, Wong L, Kennedy C, et al. Urinary soluble CD163 in active renal vasculitis. J Am Soc Nephrol. 2016;27:2906-2916.

22. Caspari T. Checkpoints: how to activate p53. Curr Biol. 2000;10: R315-R317.

23. Podhorecka M, Skladanowski A, Bozko P. H2AX phosphorylation: its role in DNA damage response and cancer therapy. J Nucleic Acids. 2010;2010:9.

24. Xiao H, Heeringa P, Hu P, et al. Antineutrophil cytoplasmic autoantibodies specific for myeloperoxidase cause glomerulonephritis and vasculitis in mice. J Clin Invest. 2002;110:955-963.

25. Al-Ani B, Fitzpatrick M, Al-Nuaimi H, et al. Changes in urinary metabolomic profile during relapsing renal vasculitis. Sci Rep. 2016;6: 38074. 